\title{
The effects of cue-target uniqueness on cued recall performance
}

\author{
BARRY STEIN \\ Vanderbilt University, Nashville, Tennessee 37240
}

\begin{abstract}
The uniqueness of the cue-target word encoding episode was examined as an important determinant of retrieval-cue effectiveness. Using a single list of unrelated word pairs, the uniqueness of the cue-target word relationship in each word pair was manipulated through two sets of encoding instructions. One set of instructions led the subjects to relate the items in each word pair in a unique way by having them treat the word pairs as similes. The other set of instructions led subjects to relate the items in each word pair in a similar way by having them compare the items in each pair with respect to their hardness properties. Subjects receiving the simile encoding instructions performed significantly better on a cued recall test, where one word from each pair was used as a cue for the other member. The encoding instructions were also found to have a significant effect on the ability to recognize word pairs, but were found to have little effect on subjects' ability to recall and recognize individual list items. Implications of the results for theories of memory are discussed.
\end{abstract}

In recent years, the investigation of factors that influence retrieval-cue effectiveness has received increased attention. This concern with factors that constrain a retrieval cue's effectiveness emanates in part from the strong implications these findings can have for theories and models which deal with the structure of memory and related retrieval processes.

One theoretical framework which has attempted to deal with the problem of retrieval-cue effectiveness is described in the encoding specificity literature (e.g., Tulving \& Thomson, 1971, 1973; Wiseman \& Tulving, 1976). Although the general theoretical position has been stated in several different forms, two important points reappear in discussions of retrieval-cue effectiveness. One point the theory makes is that a retrieval cue will be effective to the extent that it was initially encoded with the target item. At this level, the mere pairing of a cue and its target during acquisition seems sufficient to predict its effectiveness. At another point, however, the theory indicates that a retrieval cue will be effective to the extent that it precisely matches or reinstates the initial encoding of the target word. Can a cue that is encoded with the target item not precisely match the initial encoding? Tulving and his associates have demonstrated recognition failure for the target item itself in a new context. Since an exact copy of the word to be recognized may fail to reinstate the initial encoding episode, it seems very plausible that a retrieval cue also encoded with that target word could fail in reinstating the initial encoding episode when it appears in a new context.

This research was supported in part by Grants 5-TO1MNO8527-12 and NE-GOO-3-0026. The author wishes to thank Jeffery J. Franks and John D. Bransford for helpful comments on an earlier version of the manuscript.
The encoding specificity literature has only partially dealt with the specification of factors that affect the ability of a cue that is encoded with the target word to reinstate precisely the initial encoding episode involving the target item. One approach for dealing with this problem entails an analysis of the structural properties of the acquisition event in which the cue and its intended target word are embedded.

Wiseman \& Tulving (1976) have recently pointed out that, in order for the target item to be retrieved, the cue information must appropriately match the trace of the item in context. Consider an acquisition list with only one word pair. In that situation, it is doubtful that one would find any difference in recall performance by giving the first word of the pair as a retrieval cue or by giving the instructions to "recall the second word of the pair" as a retrieval cue. Both cues serve to reinstate the context of the initial encoding event in which the single target word occupies a unique role. When additional word pairs are added to the acquisition list, the effectiveness of a cue for its intended target word will not depend as much on the extent to which that cue specifies the general context of the acquisition event as on the extent to which the cue uniquely specifies or reinstates the context of the smaller encoding episode involving the to-beremembered word. It is proposed here that the ability of a cue to reinstate precisely the initial encoding of a target word depends on the uniqueness of the encoded relationship between the intended cue and target in the global structure of the encoded event.

One method of manipulating the uniqueness of cue-target relationships in the encoded acquisition event is by pairing the same cue with a number of different target items in the acquisition list. Moscovitch and Craik (1976) utilized this kind of manipulation 
and found significant effects on retrieval-cue effectiveness in semantic encoding tasks (see also Tulving \& Pearlstone. 1966). The experiments described below attempt to expand the generality of the concept of uniqueness by demonstrating effects on retrieval-cue effectiveness that are not predictable from the structure of the acquisition list used (i.e.. manipulations of the number of target words paired with a particular cue). The experiments will attempt to explore "uniqueness" effects when the cue and target pairs are unrelated across the acquisition list. The uniqueness of cuetarget relationships will be manipulated by the use of encoding instructions that either degrade or enhance the uniqueness of individual word-pair encoding episodes with respect to the information encoded in the list event. The effect of this encoding manipulation on cued recall performance. recognition performance. and free recall performance will be examined.

\section{EXPERIMENT 1}

The effectiveness of a retrieval cue encoded with its intended target word was examined in two types of encoding situations. One encoding situation required subjects to rate word pairs (e.g., bumper-statue) presented in the form. "A bumper is like a statue." as good or bad similes. The other encoding situation required subjects to rate the same word pairs presented in the form described above as similar or different with respect to their referents' hardness or softness properties. It was hypothesized that the greater uniqueness of the cue-target word relationships established in the "simile" encoding condition would promote a higher level of retrieval-cue effectiveness.

\section{Method}

Subjects. Forty undergraduates from the introductory psychology course at Vanderbilt Liniversity received course credit for their voluntary participation.

Materials. Twenty-four pairs of concrete words selected to form equal numbers of pairs of easy and difficult similes. equal numbers of pairs rated on the "hard-soft" dimension as same or different, and selected to be as homogeneous as possible with respect to frequency were used in all acquisition phases of the experiment. The word pairs were presented in the form "An $X$ is like a $Y . "$

Procedure. A two-group between-subjects design was used. Incidental learning instructions were given to both groups. Subjects were told that the purpose of the experiment was to obtain normative data that would indicate whether certain relationships between words are commonly perceived by people. During the acquisition phase. one group of subjects was instructed to rate the two words in each pair to be presented as "same" or "different" on the dimension of hard-soft by putting an appropriate mark on an answer sheet after each pair was presented. The other group of subjects was instructed to rate the words to be presented as "easy" or. "difficult" similes by putting an appropriate mark on the answer sheet after each pair was presented. Subjects in both groups were presented the 24 word pairs in the form "An $X$ is like a $Y$," with an interstimulus interval (ISI) of $2.5 \mathrm{sec}$ (pilot data indicated that an ISI of $2.5 \mathrm{sec}$ provided sufficient time for each rating task).
Following acquisition, both groups of subjects were tested on cued recall. Subjects were instructed to recall on the answer sheets the second word of the pair presented during acquisition for each first word they were given. The 24 randomized cues were presented with an ISI of $4 \mathrm{sec}$

\section{Results and Discussion}

Subjects in the hard-soft encoding situation recalled a mean of 5.24 correct target words per subject for the list of retrieval cues provided. Subjects in the simile encoding situation, who were presented with the same list of words and cues. recalled a mean of 14.31 correct target words per subject. The effect of the encoding context was significant $[\mathrm{t}(38)=8.9 . \mathrm{p}<.001]$. A further analysis of the data was undertaken to investigate the possibility that the effect detected above was correlated with differences in the number of positive ratings during acquisition between the two groups. A conditional analysis of the cued recall performance showed that. given that the subject rated the word pair by checking the "yes" column. performance for the hard-soft encoding group was $36 \%$ correct while the simile encoding group was $78 \%$ correct. The effect of encoding context on cued recall performance was still significant $[\mathrm{t}(38)=5.93 . \mathrm{p}<.001]$.

An analysis of intralist intrusion rates revealed a mean intrusion rate of .5 items for subjects in the simile encoding condition and 1.9 items for subjects in the hard-soft encoding condition. A significantly greater number of intralist intrusions [t(38)=2.98. $\mathrm{p}<.01$ ] occurred in the hard-soft encoding condition.

The 2.5.sec response interval for acquisition ratings gave subjects sufficient time to complete each rating trial in both of the encoding conditions. Further support that meaningful relational encoding was complete under each encoding condition is provided by an analysis of the consistency of responses across subjects. Within the hard-soft encoding condition. $82 \%$ of the subjects gave the modal response. while in the simile encoding an equivalent $81 \%$ of the subjects gave the modal response.

These findings are considered initial support for the hypothesis that the uniqueness of the cue-target encoding episode in an acquisition event influences the effectiveness of retrieval cues for the recall of words paired with them in the acquisition list.

\section{EXPERIMENT 2}

The results of Experiment 1 suggest that the uniqueness of the cue-target encoding episode influences the effectiveness of the relational encoding of each word pair with respect to a cued recall task. Another way of examining the effectiveness of the relational encoding of word pairs is with a pair-recognition task. Experiment 2 examines the extent to which the uniqueness of the cue-target encoding episode influences the ability to recognize correctly word pairs from the original acquisition list. 


\section{Method}

Subjects. Forty undergraduates from the introductory psychology course at Vanderbilt University received course credit for their voluntary participation.

Materials. The same acquisition list used in Experiment 1 was used in Experiment 2. A pair-recognition list composed of 12 word pairs presented in the acquisition list and 12 word pairs formed by re-pairing the remainder of the items in the acquisition list was used in the testing phase.

Procedure. A two-group between-subjects design was used. The acquisition phase was identical to that described in Experiment 1. Following acquisition, both groups of subjects were given a pair-recognition test. Subjects were instructed to put an appropriate mark on an answer sheet for each word pair in the to-be-presented list that they recognized from the acquisition list. The nature of the foils was described to the subjects. The randomized word pairs in the recognition list were presented with an ISI of 1.5 sec.

\section{Results and Discussion}

Subjects in the hard-soft encoding condition correctly recognized a mean of 9.3 pairs per subject with a false positive rate of 1.7 pairs per subject. Subjects in the simile condition correctly recognized a mean of 11.2 pairs per subject with a false positive rate of .40 pairs per subject. A comparison of the means for correct responses was significant $[\mathrm{t}(38)=4.61, p<.001]$. A compensation for false positive rates would enhance this difference in recognition performance between the hard-soft group and the simile group.

These results indicate that the uniqueness of the cue-target encoding episode affects the ability to correctly recognize word pairs from the original acquisition list. The findings of Experiments 1 and 2 suggest that the uniqueness of the encoded relationship between words in the acquisition list affects subjects' ability to detect and utilize certain structural relationships (i.e., word pairings) between words encoded contiguously in the acquisition event.

\section{EXPERIMENT 3}

The third experiment attempted to investigate one possible locus for the effect found in Experiment 1 by examining the nature of the information acquired by subjects in each of the two encoding situations. The differences in cued recall performance found in Experiment 1 and attributed to the uniqueness of cuetarget relationships may in fact be due to differences in the degree of semantic processing accompanying the encoding instructions (e.g., Arbuckle \& Katz, 1976; Craik \& Lockhart, 1972; Craik \& Tulving, 1975). If differences in the levels of processing are responsible for the results obtained in Experiment 1, one would expect to find evidence of the differences in the subjects' ability to recognize and recall individual list items (e.g., Craik \& Tulving, 1975). Experiment 3 compares recognition performance on individual items from the acquisition list for subjects in each of the two encoding conditions.

\section{Method}

Subjects. Forty undergraduates from the introductory psychology course at Vanderbilt University received course credit for their voluntary participation.

Materials. The same acquisition list used in Experiment 1 was used in Experiment 3. A recognition list composed of the 48 items in the acquisition list and 48 unrelated new words was used in the testing phase. The new words were selected to be equivalent in frequency and imagibility to the old words.

Procedure. A two-group between-subjects design was used. The acquisition phase was identical to that described in Experiment 1. Following acquisition, both groups of subjects were given a recognition test. Subjects were instructed to put an appropriate mark on the answer sheet for each word in the to-be-presented list that they recognized from the acquisition list. The randomized words in the recognition list were presented with an ISI of $1.5 \mathrm{sec}$.

\section{Results and Discussion}

Subjects in the hard-soft encoding condition had a mean recognition rate of 43.3 items per subject with a mean false positive rate of 5.7 items per subject. Subjects in the simile encoding condition had a mean recognition rate of 42.0 items per subject with a mean false positive rate of 3.8 items per subject. A comparison of the mean recognition rates was not significant $[\mathrm{t}(38)=1.56, \mathrm{p}>.10]$. Adjusting the recognition rates for false positive differences would have lessened the difference between means. It was also noted that recognition performance on the target words was equivalent to recognition performance on the cue words in both groups. The encoding context manipulation appears to have no effect on the ability to recognize individual items from the acquisition list.

\section{EXPERIMENT 4}

Experiment 4 further investigated possible differences in the nature of the information acquired by subjects in each of the two encoding conditions by comparing free recall performance for individual list items across the two encoding conditions.

\section{Method}

Subjects. Forty undergraduates from the introductory psychology course at Vanderbilt University received course credit for their voluntary participation.

Procedure. A two-group between-subjects design was used. The acquisition phase was identical to that described in Experiment 1. Thirty seconds following acquisition, both groups of subjects were given a free recall test. Subjects were instructed to write on the answer sheet as many of the target words from the acquisition list as they could remember. Three minutes were allowed for this task.

\section{Results and Discussion}

Subjects in the hard-soft encoding situation recalled a mean of 14.90 correct items per subject. Subjects in the simile encoding situation recalled a mean of 14.16 correct items per subject. A comparison of the means was not significant $[t(38)=.62, p>.10]$. 
An analysis of the kind of items recalled indicated that subjects in the simile encoding condition recalled more pairs from the acquisition list than did subjects in the hard-soft encoding condition, with means of 5.4 pairs and 4.38 pairs, respectively; however, this difference was not significant $[\mathrm{t}(38)=1.34, \mathrm{p}>.10]$. Subjects in the hard-soft encoding condition did recall more single items from the original acquisition list word pairs than did subjects in the simile encoding condition, with means of 5.95 items and 3.16 items, respectively; this difference was significant $[\mathrm{t}(38)=3.36$, $p<.01]$. These results indicate that, although the encoding instructions had no effect on the overall level of free recall, they did have some influence on the kind of items recalled.

\section{GENERAL DISCUSSION AND CONCLUSIONS}

The preceding experiments explored the effects of manipulating the uniqueness of the intended cue-target relationship in the acquisition event. The results of Experiments 1 and 2 demonstrated that the simile encoding instructions, which led to the encoding of more unique cue-target relationships across word pairs, yielded more effective retrieval cuing and pairrecognition performance. Experiments 3 and 4 explored the locus of the uniqueness effect. Both encoding tasks yielded equivalent performance levels in situations where subjects were tested about specific items presented and not about relations between items.

The results demonstrate that one factor which determines whether a retrieval cue encoded together with a target item will be effective in precisely reinstating the initial encoding of the target word is the uniqueness of the relationship between the cue and target. The results also suggest a broader context for defining the uniqueness effects demonstrated by Moscovitch and Craik (1976). In their study, uniqueness effects on cued recall performance were obtained by manipulating the number of target words paired with a particular kind of cue. The uniqueness of the cuetarget relationship was identified with the similarity of cues in the structure of the acquisition list. The experiments performed here suggest that the uniqueness of a given cue-target relationship is a function of the encoded relationships between cues and targets and this is not necessarily reflected in the nominal structure of the acquisition list.

\section{REFERENCES}

ARbuCKLE, T. Y., \& KATZ, W. A. Structure of memory trace following semantic and non-semantic orientation tasks in incidental learning. Journal of Experimental Psychology: Human Learning and Memory, 1976, 2, 362-369.

Craik, F. I. M., \& LockharT, R. S. Levels of processing: A frame-work for memory research. Journal of Verbal Learning and Verbal Behavior, 1972, 11, 671-684.

Craik, F. I. M., \& Tulving, E. Depth of processing and the retention of words in episodic memory. Journal of Experimental Psychology: General, 1975, 104, 268-294.

Moscovitch, M., \& Craik, F. I. M. Depth of processing, retrieval cues, and uniqueness of encoding as factors in recall. Journal of Verbal Learning and Verbal Behavior, $1976,15,447-458$.

Tulving, E., \& Pearlstone, $Z$. Availability versus accessibility of information in memory for words. Journal of Verbal Learning and Verbal Behavior, 1966, 5, 381-391.

Tulving, E., \& Thomson, D. M. Retrieval processes in recognition memory: Effects of associative context. Journal of Experimental Psychology, 1971. 87, 116-124.

Tulving, E., \& THOMson, D. M. Encoding specificity and retrieval processes in episodic memory. Psychological Review, 1973, 80, 352-373.

Wiseman, S.. \& Tulving, E. Encoding specificity: Relation between recall superiority and recognition failure. Journal of Experimental Psychology: Human Learning and Memory, 1976, 2, 349-361.

(Received for publication November 2, 1976; revision accepted January 27, 1977.) 